\title{
A Combined Color and Texture Features Based Methodology for Recognition of Crop Field Image
}

\author{
M. V. Latte ${ }^{1}$, Sushila Shidnal ${ }^{2}$, B.S. Anami ${ }^{3}$ and V B Kuligod ${ }^{4}$ \\ ${ }^{1}$ Principal JSSATE, Bangalore, ${ }^{2}$ Assistant Professor, SMVIT, Bangalore \\ ${ }^{3}$ Principal KLEIT, Hubli, ${ }^{4}$ Professor UAS, Dharwad \\ mvlatte@rediffmail.com,sushram.shidnal@gmail.com, \\ anami_basu@hotmail.com,vbkuligod@yahoo.co.in
}

\begin{abstract}
This paper presents a methodology to recognize certain crop fields' images using texture, color and combination of both types of features. In this work, we have considered eight varieties of crop images, namely, Brinjal, Cotton, Groundnut, Paddy, Soyabean, Sugarcane and Sunflower. Texture features using GLCM and color features using HSV are deployed. Artificial Neural Network (ANN) is used for recognition. Considering only as feature, classification accuracies of $63.75 \%, 66.25 \%$ and $84.375 \%$ are obtained using texture, color and their combination respectively. The work is helpful in the area of agriculture for early detection and prevention of diseases.
\end{abstract}

Keywords: Field images, GLCM, HSV, Artificial Neural Network and Pattern recognition.

\section{Introduction}

Agriculture is the major source of income for about three-fourths of India's rural population. Technological support to Agriculture is considered important in assessing the development of any country. Poorly trained farmers cannot apply the precision methods and new technologies like use of robots or the use of new irrigation techniques or use of sensors to predict the yield etc. Common technological applications like human computer interaction, virtual environment, robotics and multimedia, include computer vision, image processing and speech processing are possible in the field of Agriculture. Computer vision helps in analyzing the visual input from an image and produces a description to interact with the world. In the proposed work, eight types of fields' images, namely, Cotton, Sugarcane, Paddy, Sunflower, Brinjal, Soyabean, Maize and Groundnut are considered and some sample images are shown in Fig. 1.
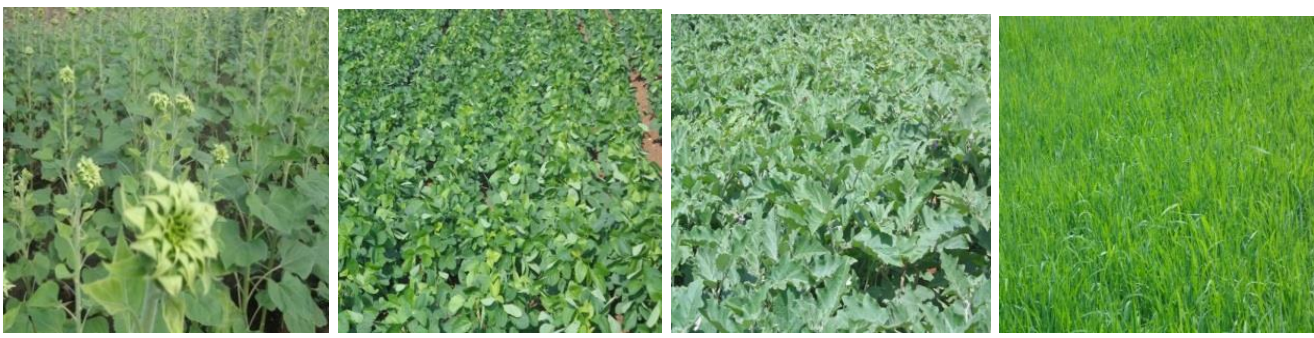

Figure 1. Images of Sunflower, Soyabean, Brinjal and Paddy Fields

Two types of features, namely, texture and color are extracted. Texture features are extracted using Gray level co-occurrence matrix (GLCM) and color features from HSV model. These extracted features are used to train and test ANN as recognizer. In order to 
know the state- of-the-art development in automation activities in the fields of agriculture and horticulture, we have carried out a literature survey. The gist of the literature survey is given in this section 2.0.

\section{Literature Survey}

Hermawan syahputra, et al, (2014) have proposed a plant recognition system of stereo leaf images using gray level co-occurrence matrix. The methodology has used sum of absolute difference (SAD) and 22 features are extracted using GLCM. The accuracy reported is $83.3 \%$. Pallavi P et al, (2014) have proposed leaf recognition based on Zernike moments. This methodology uses shape, vein, color and texture features to identify the leaves. A neural network approach is used to classify the images. Gowri Ariputhiran, et al, (2013) have proposed feature extraction and classification of high resolution satellite images using GLCM and back propagation technique. The performance is analyzed based on its accuracy, error rate and sensitivity. Vishaka Metre et al, (2013) have proposed an overview of the research on texture based plant leaf classification. In this method a leaf is classified based on different morphological features. Manish Maheshwari et al, (2013) have proposed a new feature extraction technique for color image clustering. A color quantization ordering scheme that focuses on color as feature and considers Hue, Saturation and Value space is proposed. To form clusters of images k-means algorithm is applied.

A H kulkarni, et al,(2013) have proposed a framework for recognizing and identifying plants using various features, combined with zernike movements. Classifier used is radial basis probabilistic neural network (RBPNN). Dual stage training algorithm is used to train RBPNN to increase performance of the classifier. Flavia leaf is used for the study. The experimental simulation results show an accuracy rate of $93.82 \%$. Smita Naikwad, et al, (2013) have given an account of advances in image processing for detection of plant diseases using color and texture features. The technique has detected and classified the plant diseases with an accuracy of $83 \%$. S.Abhirami, et al,(2012) have proposed plant species identification based on images of flowers. The features extracted are shape, color and texture. Color based segmentation is carried out using k-means algorithm and active contour model. Texture features are extracted using GLCM. Edge detection is done using Sobel, Robert and Prewitts operators. The classification is done using proximal support vector machine. Abdul Kadir, et al, (2012) have proposed foliage plan identification based on leaves using zerkine moments. Zernike moments are combined with other features like geometric features, color moments and gray level co-occurrence matrix. For identification, probabilistic neural network is used. The experiment show that combined features yield good result.

Ashutosh Kumar Bhatt et al, (2012) have proposed an artificial neural network based apple classifier. Input is collected from software and web camera, ANN is used to classify apple according to quality. Experimental data show better results compared to predicted values. Dr J Abdul Jakeel et al, (2012) have proposed an artificial neural network based detection of skin cancer. The methodology for feature extraction used is $2 \mathrm{D}$ wavelet transform. ANN is used as a classifier. Norasyikin Fadilah et al,(2012) have proposed oil palm fresh fruit bunch ripeness classification using ANN. This method is used to automate the decision of grading oil palm FFBs (fresh fruit bunches), which replaces manual human grading method. Results show that ANN is able to generalize four ripeness categories of oil palm FFB. R Venkta Ramana Chary, et al, (2012) have proposed feature extraction method for color image similarity. Color projection and different mathematical approaches like mean, median, standard deviation are applied to retreive images within a large collection of images. For retreival of images, RGB color combinations are considered. Results are observed to be efficient.

Haipeng Yu, et al,(2009) have proposed image retrieval method which includes color, textural and special features of wood species. A total of 9 features are extracted to retrieve 
wood species. The results show that combined effect of these features yield good results. LiyingZheng, et al,(2008) have proposed a method to separate green vegetation in color images using mean shift procedure for segmentation. Multiple color features are extracted like hue, saturation in HSI color space and red, green, blue in RGB space. With mean shift segmentation algorithm, extracted features and BPNN, images are classified as green and non-green. R Pyadipati, et al,(2006) have proposed a color co-occurrence method $(\mathrm{CCM})$ and discriminant analysis method to recognize citrus plant diseases. The method used texture based Hue, Saturation and Intensity (HSI) color features extracted using CCM and statistical classification algorithm to identify diseased and normal citrus leaves. $\mathrm{H} \mathrm{Fu}$ and $\mathrm{Z} \mathrm{Chi}$, (2006) have proposed a combined approach of using artificial neural network and threshold to recognize plants based on images. Leaf veins are extracted to define features for plant species recognition. The results show that combined approach is good.

From the literature survey, it is observed that certain work on recognition of plants based on leaf characteristics has already been carried out. Several feature extraction methods like vein, Zernike moments etc. are being deployed. However, no considerable work is carried out on recognition of field images of varieties of crops. Human beings rarely view one or two leaves in a field. Instead, the entire field image is considered for recognition. This is the motivation for the proposed work.

The remaining part of the paper is organized into four sections. Section two gives the literature survey, section three deals with methodology, which further explain about image acquisition, preprocessing, feature extraction and classification, section four gives with results and discussion and finally section five deals with conclusion.

\section{Methodology}

The proposed methodology on combined color and texture features based methodology for classification of crop field images is divided into four steps, namely, Image acquisition, Preprocessing, Feature extraction and Classification as shown in Fig. 2.

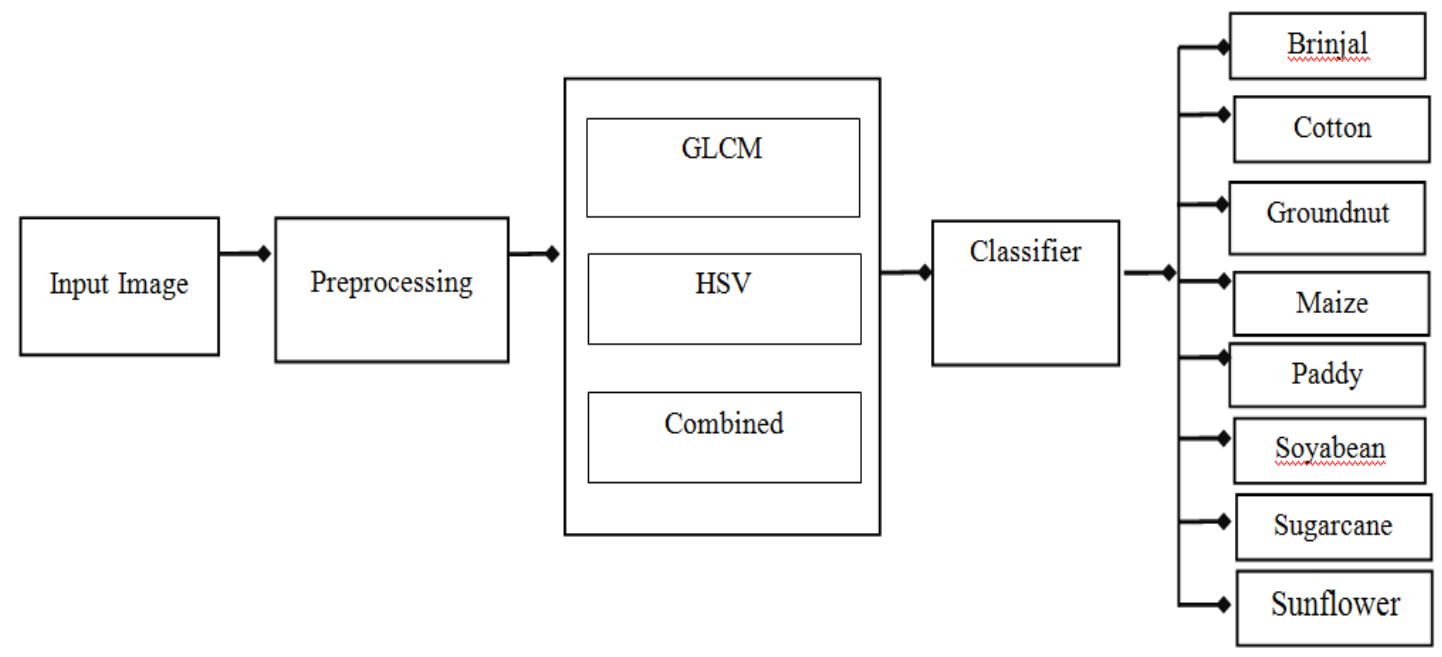

Figure 2. Block Diagram of the Proposed Work

\subsection{Image Acquisition}

The field's images are captured using Sony digital camera of 16.1 Mega Pixels. The images are captured under fixed focal length under standard illumination conditions. The field images of Paddy, Sugarcane, Sunflower, Groundnut, Cotton, Maize, Soya Bean, and 
Brinjal are used in the present work. The data set consists of 20 images of each field crop. A total of 180 images are considered for experimental study. Some of the sample images are as shown in the Fig. 3.
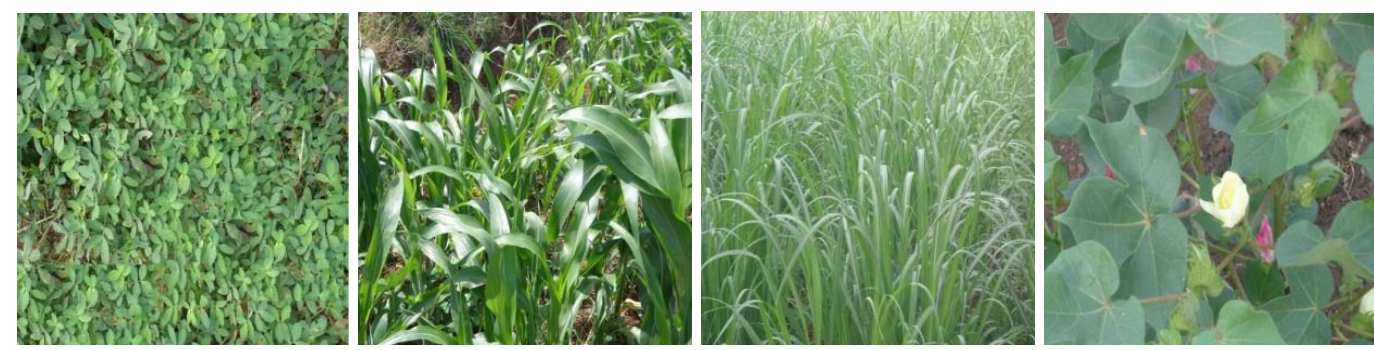

Figure 3. Field images of Groundnut, Maize, Sugarcane and Cotton

\subsection{Preprocessing}

The fields' images captured are of 3456 X 4608 pixels. We have filtered and resized the images. Median filter is found to be suitable for the proposed work. The images are resized to 512 X 512 pixels. A sample image of paddy field before and after filtering is shown in Fig. 4.
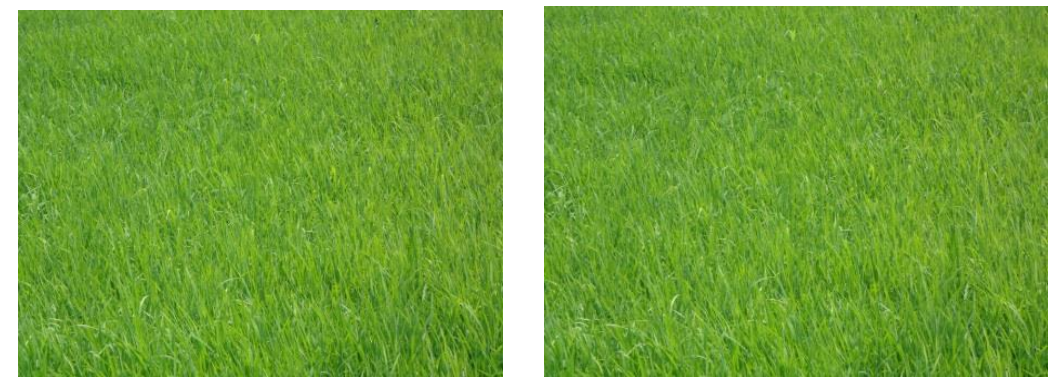

Figure 4. Sample Image before and After Filtering

\subsection{Feature Extraction}

From literature survey, we found that feature extraction techniques used are color, texture, shape etc. In the proposed methodology, an exhaustive experimentation is carried out considering color and texture features.

3.3.1 Gray Level Co-occurrence Matrix (GLCM): Visual system of human beings use second order distribution of gray levels as discriminator in identifying textures. Some of the characteristics of texture are homogeneity, entropy, contrast and others. GLCM is very useful to obtain valuable information about the relative position of the neighboring pixels in an image. The co-occurrence matrix GLCM (i,j) counts the co-occurrence of pixels with gray value $i$ and $j$ at given distance $d$. The matrix element $P(i, j)$ is separated from its neighborhood by a pixel distance $(\Delta x, \Delta y)$, one with intensity I and the other with intensity j. Number of gray levels is denoted by G. ${ }^{\mu}$ is the mean value of P. ${ }^{\mu_{\mathrm{x}}}$ and $\mu_{y}$ are the means and standard deviations of Px and Py. The direction of neighboring pixels to represents the distance can be selected, for example $135 \mathrm{o}, 90 \mathrm{o}, 45 \mathrm{o}$, or $0 \mathrm{o}$, as illustrated in Figure 5. 


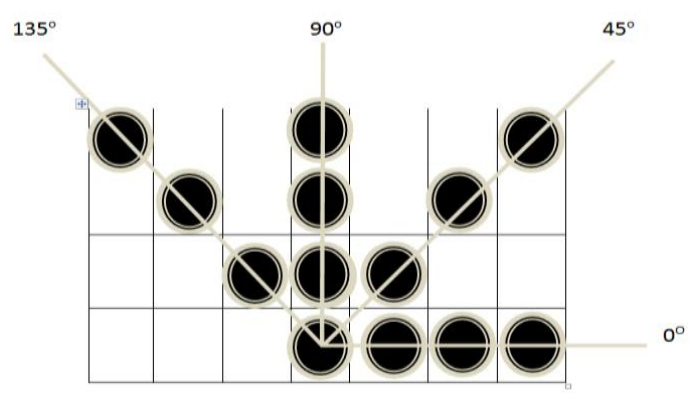

Figure 5. Directions in Calculating GLCM

Autocorrelation $=\sum_{i} \sum_{j}(i j) \cdot P(i, j)$

Table 1. Autocorrelation Feature Values for Different Field Images

\begin{tabular}{|l|l|l|l|l|l|l|c|}
\hline Brinjal & Cotton & $\begin{array}{c}\text { Groundnu } \\
\mathrm{t}\end{array}$ & Maize & Paddy & $\begin{array}{c}\text { Soyabea } \\
\mathrm{n}\end{array}$ & Sugarcane & Sunflower \\
\hline 24.1119 & 22.0856 & 22.5134 & 23.2861 & 21.1201 & 20.7113 & 24.7828 & 19.7109 \\
\hline 23.6060 & 29.6321 & 20.9259 & 21.8625 & 20.6094 & 21.7553 & 25.3469 & 20.7545 \\
\hline 24.3305 & 23.5454 & 19.3553 & 19.5751 & 19.5246 & 20.1003 & 23.2533 & 21.0163 \\
\hline 24.2179 & 20.8160 & 24.8980 & 24.2241 & 20.6673 & 19.8544 & 24.1300 & 21.8298 \\
\hline 22.3906 & 21.0447 & 21.5332 & 24.3650 & 21.3370 & 20.5203 & 21.2734 & 20.9112 \\
\hline 20.1065 & 21.5781 & 20.7822 & 22.8061 & 19.5573 & 21.8742 & 26.8223 & 28.1540 \\
\hline 22.8760 & 19.7231 & 19.1834 & 12.8416 & 19.2251 & 19.9482 & 22.5949 & 24.0996 \\
\hline 21.4424 & 24.5872 & 22.6780 & 13.6716 & 19.9423 & 22.2696 & 26.0679 & 24.5178 \\
\hline 24.5491 & 27.5046 & 21.5045 & 26.0302 & 22.4673 & 21.9306 & 26.7388 & 22.6717 \\
\hline 25.3675 & 22.4095 & 21.7652 & 23.6258 & 22.1956 & 22.6315 & 25.1625 & 22.2957 \\
\hline 23.8400 & 23.0675 & 20.6994 & 24.5443 & 23.1584 & 19.6667 & 24.3797 & 22.3222 \\
\hline 24.4419 & 21.4232 & 21.9096 & 21.1245 & 22.1867 & 23.0422 & 24.5458 & 22.0486 \\
\hline 25.1366 & 23.6985 & 22.7019 & 22.9797 & 21.4486 & 21.8950 & 25.1323 & 24.1313 \\
\hline 24.2559 & 20.4671 & 23.4563 & 24.4630 & 21.7487 & 23.2932 & 22.1501 & 23.7007 \\
\hline 23.3021 & 23.9773 & 22.9697 & 25.1126 & 21.1201 & 20.5436 & 21.8302 & 21.5769 \\
\hline 24.1588 & 25.1650 & 21.1602 & 17.0398 & 20.6094 & 23.1840 & 21.8271 & 20.7545 \\
\hline 22.2288 & 24.9027 & 22.6895 & 22.7387 & 19.5246 & 21.1492 & 25.5935 & 19.7109 \\
\hline 22.1743 & 22.1374 & 22.5410 & 21.4434 & 20.6673 & 22.4197 & 26.1134 & 21.6968 \\
\hline 22.9865 & 24.6083 & 23.7960 & 19.8601 & 22.1956 & 23.0956 & 27.7082 & 28.1540 \\
\hline 22.2812 & 24.1615 & 23.7960 & 24.6506 & 23.1584 & 25.1228 & 24.4146 & 24.0996 \\
\hline
\end{tabular}

In the proposed work, we have considered all the 22 features, initially. The five prominent texture features, namely, autocorrelation, cluster prominence, sum of squares of variance, sum of variance and sum of average are utilized in the work. The reduction in the number of features Autocorrelation refers to repeating patterns like presence of periodic signal obscured by noise and is given by equation (1). The autocorrelation values for the different varieties of field images are given in Table 1. Considering the tabular values the classification accuracy of the extracted feature autocorrelation is as shown in the Fig.6. 


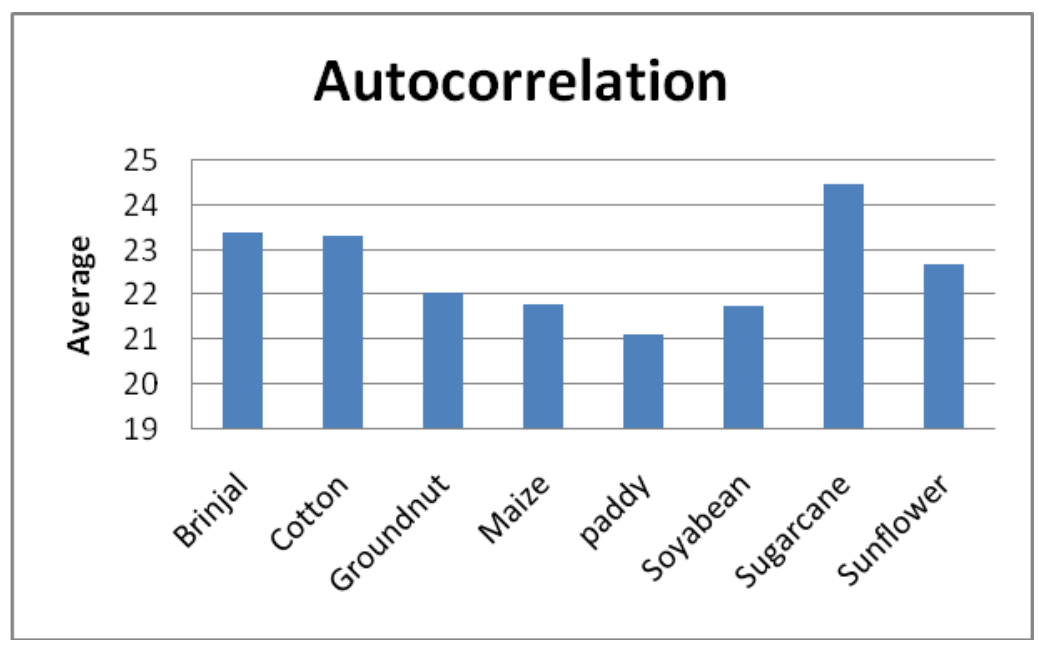

Figure 6. Autocorrelation Classification Accuracy

Cluster prominence is the measure of the skew in of the matrix, in other words the lack of symmetry and is given by the equation (2). The extracted feature values from the algorithm are given in the Table2. The classification accuracy of cluster prominence is given in the Fig.7.

$$
\text { Cluster Prominence }={ }^{\sum_{i}} \sum_{j} P(i, j) \cdot(i-\mu x+j-\mu y)_{4}
$$

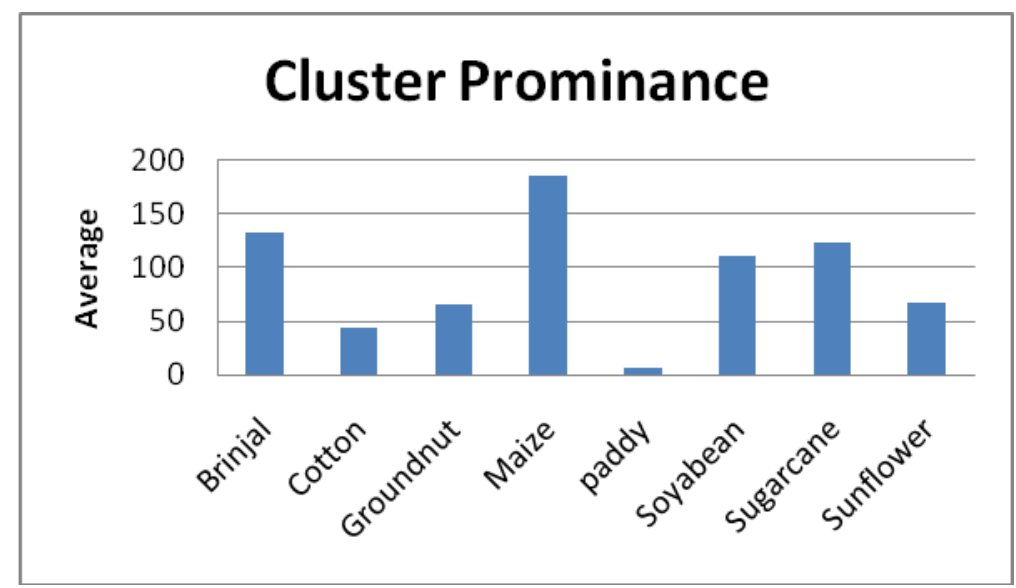

Fig.7. Cluster Prominence Classification Accuracy

Table 2. Cluster Prominence Values of all Field Images

\begin{tabular}{|c|c|c|c|c|c|c|c|}
\hline Brinjal & Cotton & Groundnut & Maize & Paddy & Soyabean & Sugarcane & Sunflower \\
\hline 130.2619 & 18.0145 & 54.3874 & 264.3967 & 9.5720 & 112.4264 & 56.0695 & 66.7312 \\
\hline 168.6870 & 52.2004 & 44.8960 & 199.3252 & 1.6932 & 121.6650 & 197.6057 & 31.1979 \\
\hline 157.7387 & 63.6262 & 45.5494 & 158.2652 & 5.5684 & 144.1989 & 49.0297 & 62.9901 \\
\hline 236.3847 & 15.2933 & 23.5871 & 253.9035 & 2.6715 & 176.8790 & 28.6825 & 49.9067 \\
\hline 281.9142 & 36.8663 & 32.4154 & 147.1910 & 15.5241 & 44.4060 & 20.7930 & 64.4407 \\
\hline 33.2573 & 31.0668 & 26.1112 & 241.0088 & 31.4437 & 60.7674 & 285.6442 & 153.4413 \\
\hline
\end{tabular}




\begin{tabular}{|c|c|c|c|c|c|c|c|}
\hline 37.5427 & 24.2235 & 30.7968 & 88.6757 & 2.9750 & 61.2150 & 32.4268 & 46.6054 \\
\hline 34.2149 & 11.1889 & 18.4072 & 86.2144 & 3.4638 & 40.6355 & 255.7799 & 200.0652 \\
\hline 71.0562 & 21.3162 & 71.3125 & 405.0479 & 5.5096 & 82.0314 & 337.7018 & 67.1831 \\
\hline 61.2947 & 30.9508 & 37.2266 & 193.2681 & 3.6783 & 51.7179 & 230.6312 & 30.2503 \\
\hline 84.9212 & 106.6059 & 50.1306 & 121.6926 & 4.3150 & 201.5391 & 435.1584 & 43.5750 \\
\hline 117.6614 & 12.9524 & 66.1265 & 126.7949 & 2.9960 & 109.7506 & 104.3095 & 38.9869 \\
\hline 78.5932 & 9.6271 & 129.2786 & 198.1362 & 12.0614 & 109.3417 & 44.4894 & 95.4558 \\
\hline 235.9950 & 10.0365 & 97.0550 & 241.5461 & 4.4007 & 121.0959 & 40.6179 & 38.1755 \\
\hline 219.7504 & 86.3774 & 86.5048 & 177.9869 & 9.5720 & 87.1005 & 37.0213 & 46.7128 \\
\hline 162.7342 & 73.5733 & 23.6529 & 250.2582 & 1.6932 & 129.6857 & 72.1816 & 31.1979 \\
\hline 175.3604 & 56.0388 & 126.2489 & 149.7133 & 5.5684 & 78.8703 & 27.9132 & 66.7312 \\
\hline 164.5568 & 27.8576 & 146.5334 & 28.9316 & 2.6715 & 91.0222 & 25.8416 & 22.2182 \\
\hline 102.3618 & 62.1862 & 94.8570 & 188.3717 & 3.6783 & 183.6837 & 138.8371 & 153.4413 \\
\hline 94.4015 & 131.5426 & 94.8570 & 187.5685 & 4.3150 & 225.3045 & 37.5815 & 46.6054 \\
\hline
\end{tabular}

Sum of squares of variance is the sum of squared differences from the mean and is given by equation (3).

$$
\text { Sum of squares of variance }=\sum_{i=0}^{G-1} \sum_{j=0}^{G-1}(i-\mu)^{2} \text { i } \mathrm{P}(\mathrm{i}, \mathrm{j})
$$

The values of the sum of squares of variances (sosvh) are given in Table 3. The classification accuracy for sum of squares of variance feature is as shown in the Fig. 8 .

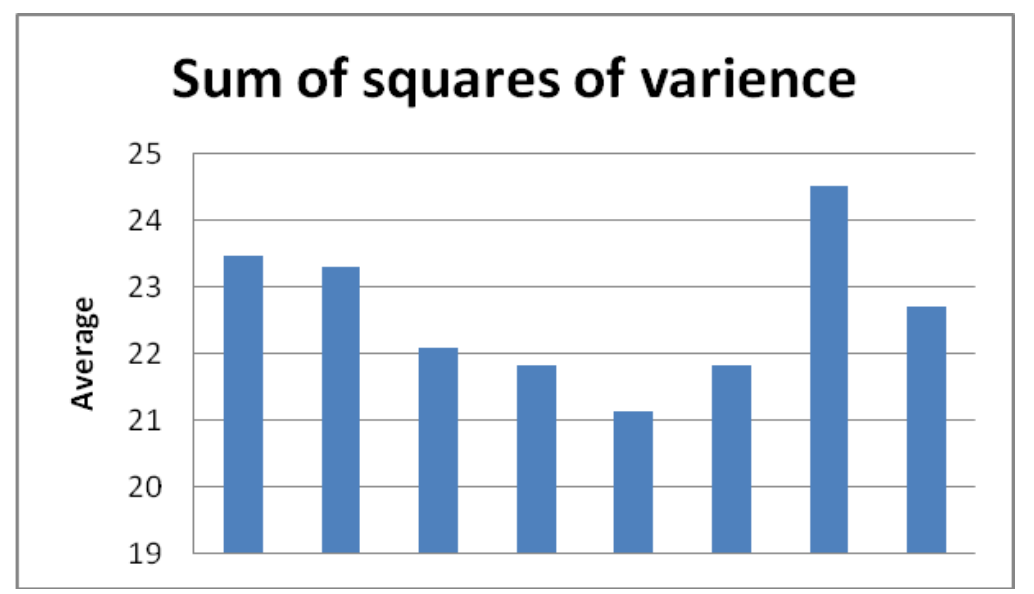

Fig 8 Sum of Squares of Variance Classification Accuracy 
Table 3. Sum of Squares of Variance Values of all Field Images

\begin{tabular}{|c|c|c|c|c|c|c|c|}
\hline Brinjal & Cotton & $\begin{array}{l}\text { Groundn } \\
\text { ut }\end{array}$ & Maize & Paddy & $\begin{array}{l}\text { Soyabea } \\
\mathrm{n}\end{array}$ & $\begin{array}{l}\text { Sugarcan } \\
\text { e }\end{array}$ & $\begin{array}{l}\text { Sunflowe } \\
\mathrm{r}\end{array}$ \\
\hline 24.1566 & 22.0228 & 22.6526 & 23.3798 & 21.1373 & 20.7586 & 24.7548 & 19.6587 \\
\hline 23.7573 & 29.6282 & 20.9673 & 21.8541 & 20.6254 & 21.7740 & 25.3031 & 20.7065 \\
\hline 24.4243 & 23.5094 & 19.3631 & 19.5800 & 19.5839 & 20.1669 & 23.2380 & 21.0450 \\
\hline 24.3550 & 20.7342 & 24.8057 & 24.2419 & 20.7086 & 19.9869 & 24.1338 & 21.8592 \\
\hline 22.5699 & 20.9620 & 21.4894 & 24.3159 & 21.3518 & 20.6390 & 21.2715 & 21.0124 \\
\hline 20.0308 & 21.5441 & 20.7263 & 22.8279 & 19.6264 & 22.0325 & 26.7849 & 28.0967 \\
\hline 22.8105 & 19.6722 & 19.1731 & 12.8579 & 19.1862 & 20.0615 & 22.5508 & 24.0397 \\
\hline 21.3863 & 24.6177 & 22.5945 & 13.6609 & 19.9331 & 22.3584 & 26.0171 & 24.6117 \\
\hline 24.6366 & 27.4719 & 21.7420 & 26.0013 & 22.4216 & 22.0703 & 26.8215 & 22.7137 \\
\hline 25.4699 & 22.3491 & 21.8990 & 23.6476 & 22.2014 & 22.7237 & 25.1151 & 22.3411 \\
\hline 23.9552 & 23.0657 & 20.7959 & 24.5192 & 23.1431 & 19.8833 & 24.4746 & 22.3181 \\
\hline 24.5059 & 21.4551 & 21.9804 & 21.2582 & 22.1690 & 23.1827 & 24.4983 & 22.1988 \\
\hline 25.1807 & 23.7255 & 22.7222 & 22.9989 & 21.4747 & 21.9486 & 25.3698 & 24.1548 \\
\hline 24.4059 & 20.4899 & 23.4881 & 24.4410 & 21.7382 & 23.2888 & 22.1174 & 23.7482 \\
\hline 23.4594 & 23.9571 & 23.0488 & 25.0653 & 21.1373 & 20.5028 & 21.8106 & 21.5856 \\
\hline 24.2877 & 25.1389 & 21.1517 & 17.0443 & 20.6254 & 23.2347 & 21.8212 & 20.7065 \\
\hline 22.4083 & 24.8271 & 22.6780 & 22.7078 & 19.5839 & 21.1409 & 25.7321 & 19.6587 \\
\hline 22.3608 & 22.1216 & 22.5560 & 21.4120 & 20.7086 & 22.4908 & 26.2482 & 21.5961 \\
\hline 23.0799 & 24.5620 & 23.8240 & 20.0217 & 22.2014 & 23.1592 & 27.8217 & 28.0967 \\
\hline 22.3022 & 24.1143 & 23.8240 & 24.6351 & 23.1431 & 25.1316 & 24.6156 & 24.0397 \\
\hline
\end{tabular}

Variance is a measure of the dispersion of the values around the mean and combinations of reference and neighbor pixels. The extracted feature Sum of variance is given by equation (4).

$$
\text { Sum of variance }=\sum_{i=1}^{2 G}(1-\mu)^{2} . \mathrm{P}(\mathrm{i}, \mathrm{j})
$$

The values of sum of variance feature (svarh) are given in Table 4 .

Table 4. Sum of Variance Values of all Field Images

\begin{tabular}{|c|c|c|c|c|c|c|c|}
\hline Brinjal & Cotton & $\begin{array}{l}\text { Groundnu } \\
\mathrm{t}\end{array}$ & Maize & Paddy & $\begin{array}{l}\text { Soyabea } \\
\mathrm{n}\end{array}$ & $\begin{array}{l}\text { Sugarcan } \\
\mathrm{e}\end{array}$ & $\begin{array}{l}\text { Sunflowe } \\
\mathrm{r}\end{array}$ \\
\hline 61.5071 & 61.0611 & 55.7271 & 56.9893 & 58.3234 & 49.3826 & 66.2892 & 50.7963 \\
\hline 58.3437 & 80.3930 & 52.0006 & 53.0085 & 61.8918 & 52.8026 & 67.1609 & 56.3565 \\
\hline 61.2019 & 63.1366 & 47.5872 & 47.3914 & 54.4222 & 46.8611 & 59.3473 & 53.3639 \\
\hline 59.4160 & 58.1708 & 70.8356 & 59.9239 & 60.6515 & 45.7124 & 63.6148 & 56.0812 \\
\hline
\end{tabular}




\begin{tabular}{|l|l|l|l|l|l|l|l|}
\hline 52.8150 & 59.9018 & 56.3524 & 61.2630 & 57.0448 & 50.6348 & 55.6125 & 51.1937 \\
\hline 55.4595 & 56.8757 & 57.5992 & 55.4469 & 49.0071 & 53.4473 & 71.5249 & 74.2299 \\
\hline 61.6839 & 52.1498 & 49.0793 & 29.3502 & 56.7187 & 47.7280 & 59.5911 & 65.6907 \\
\hline 56.8136 & 68.4345 & 64.1541 & 31.6332 & 58.0660 & 56.3497 & 70.1662 & 61.0937 \\
\hline 62.1173 & 79.7163 & 51.5087 & 66.2641 & 69.0747 & 53.0418 & 68.7146 & 57.8827 \\
\hline 65.1970 & 60.5256 & 54.9923 & 57.8917 & 66.5957 & 56.2368 & 66.6322 & 59.2300 \\
\hline 59.1702 & 58.8455 & 50.9031 & 62.3729 & 70.2538 & 44.9776 & 61.5559 & 59.4742 \\
\hline 60.6539 & 58.0381 & 54.2515 & 50.4392 & 67.0080 & 56.1474 & 65.6986 & 55.8162 \\
\hline 63.5955 & 66.1316 & 56.3702 & 57.2550 & 59.2134 & 53.5573 & 65.1201 & 62.0724 \\
\hline 59.1868 & 56.2105 & 61.1277 & 61.1419 & 64.6484 & 58.2847 & 58.1461 & 63.8025 \\
\hline 55.9458 & 64.2813 & 56.9338 & 63.1232 & 58.3234 & 51.0583 & 56.0741 & 56.5042 \\
\hline 61.1356 & 69.1056 & 55.9701 & 39.8998 & 61.8918 & 57.6098 & 54.3368 & 56.3565 \\
\hline 52.6087 & 71.0402 & 56.9390 & 59.3872 & 54.4222 & 53.2897 & 68.1268 & 50.7963 \\
\hline 52.6290 & 59.4655 & 55.8722 & 57.2754 & 60.6515 & 54.7261 & 70.1771 & 63.5427 \\
\hline 56.3020 & 67.7406 & 61.0470 & 46.1985 & 66.5957 & 55.5514 & 71.1651 & 74.2299 \\
\hline 55.4269 & 62.6439 & 61.0470 & 61.3892 & 70.2538 & 62.5564 & 62.6972 & 65.6907 \\
\hline
\end{tabular}

The classification accuracy for sum of variance feature is given in Fig. 9.

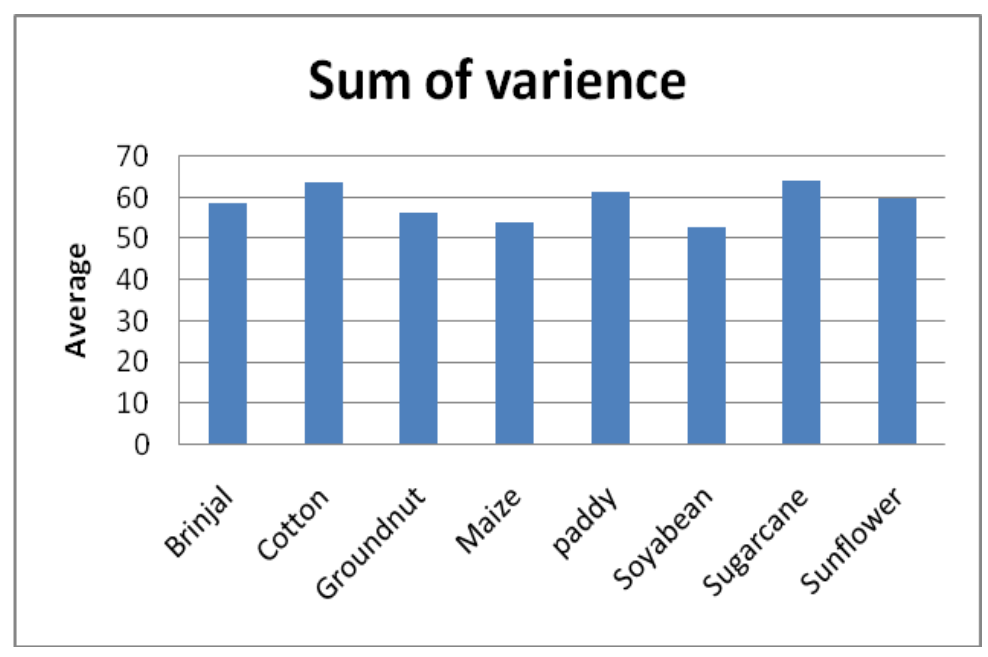

Figure 9. Sum of Variance Classification Accuracy

Sum of average is the sum of all values and divided by the total number of values and is given by the equation (5).

$$
\text { Sum of average }(\text { Mean })=\sum_{k=2}^{2 G} k \sum_{i j} P(i, j)
$$

The texture features extracted out of the above mentioned feature is given in Table 5. The classification accuracy for the sum of average feature is given Fig. 10. 


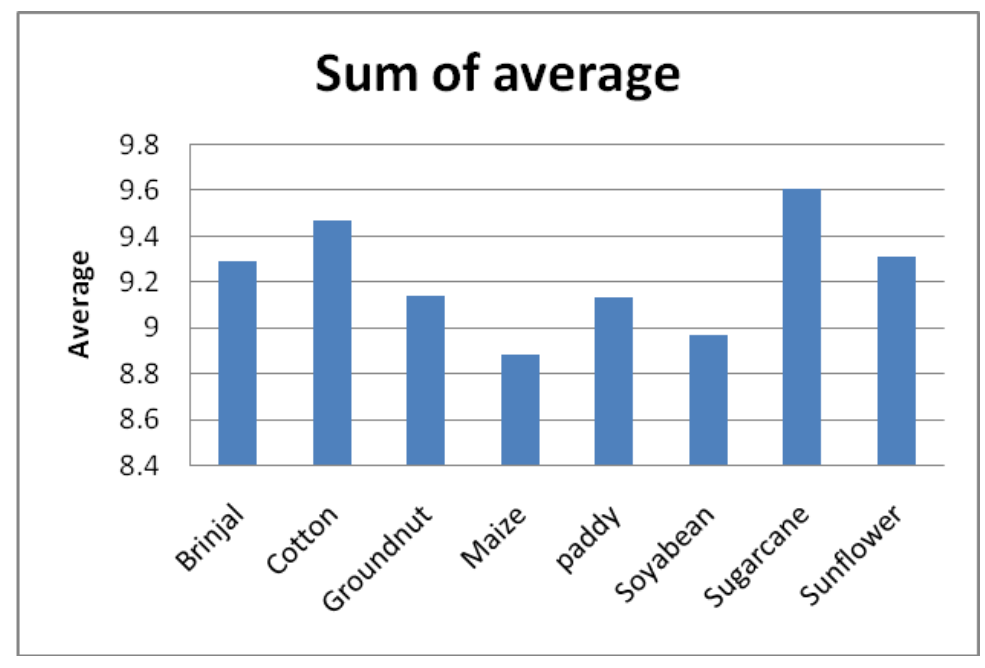

Figure 10. Sum of Average Classification Accuracy

Table 5. Sum of Average Values of all Field Images

\begin{tabular}{|c|c|c|c|c|c|c|c|}
\hline Brinjal & Cotton & $\begin{array}{l}\text { Groundn } \\
\text { ut }\end{array}$ & Maize & Paddy & $\begin{array}{l}\text { Soyabea } \\
\mathrm{n}\end{array}$ & $\begin{array}{l}\text { Sugarcan } \\
\mathrm{e}\end{array}$ & $\begin{array}{l}\text { Sunflowe } \\
\mathrm{r}\end{array}$ \\
\hline 9.4608 & 9.2669 & 9.2660 & 9.1161 & 9.1142 & 8.7629 & 9.7759 & 8.6273 \\
\hline 9.2564 & 10.6692 & 8.9258 & 8.9156 & 9.0502 & 8.9662 & 9.6955 & 8.9612 \\
\hline 9.4658 & 9.4784 & 8.5723 & 8.4805 & 8.7848 & 8.5633 & 9.4030 & 8.9623 \\
\hline 9.2511 & 9.0024 & 9.8378 & 9.3062 & 9.0597 & 8.4843 & 9.6760 & 9.1472 \\
\hline 8.8063 & 9.0416 & 9.0994 & 9.4945 & 9.1273 & 8.8666 & 9.0824 & 8.8760 \\
\hline 8.7888 & 9.1222 & 8.9754 & 9.0134 & 8.6839 & 9.1204 & 9.8525 & 10.2754 \\
\hline 9.3653 & 8.7187 & 8.5738 & 6.8589 & 8.7180 & 8.6639 & 9.3375 & 9.6517 \\
\hline 9.0722 & 9.8355 & 9.3989 & 7.0576 & 8.8817 & 9.2594 & 9.7571 & 9.5347 \\
\hline 9.6551 & 10.3755 & 9.0204 & 9.5221 & 9.4383 & 9.0964 & 9.7593 & 9.3032 \\
\hline 9.8609 & 9.2932 & 9.1657 & 9.2769 & 9.3861 & 9.2927 & 9.6139 & 9.3143 \\
\hline 9.4690 & 9.2582 & 8.8820 & 9.5890 & 9.5869 & 8.3334 & 9.1917 & 9.2822 \\
\hline 9.5250 & 9.1710 & 9.1081 & 8.8248 & 9.3831 & 9.2271 & 9.6724 & 9.2276 \\
\hline 9.7472 & 9.6609 & 9.1034 & 9.1625 & 9.1791 & 8.9598 & 9.8693 & 9.5740 \\
\hline 9.2979 & 8.9740 & 9.3833 & 9.4269 & 9.2831 & 9.2522 & 9.2431 & 9.5938 \\
\hline 9.1285 & 9.5111 & 9.3253 & 9.5670 & 9.1142 & 8.7088 & 9.1505 & 9.1247 \\
\hline 9.4326 & 9.8013 & 9.0591 & 7.7011 & 9.0502 & 9.2006 & 9.0796 & 8.9612 \\
\hline 8.9608 & 9.7750 & 9.1015 & 9.2320 & 8.7848 & 8.9070 & 9.9889 & 8.6273 \\
\hline 8.9780 & 9.2675 & 9.0337 & 9.1091 & 9.0597 & 9.1331 & 10.1008 & 9.1982 \\
\hline 9.2288 & 9.6921 & 9.4624 & 8.4695 & 9.3861 & 9.1138 & 10.2461 & 10.2754 \\
\hline 9.0843 & 9.4656 & 9.4624 & 9.4849 & 9.5869 & 9.4440 & 9.7178 & 9.6517 \\
\hline
\end{tabular}

3.3.2 Color Features: There are different color spaces such as HSV, CMY, and LUV etc. Color intensity is represented independently in HSV (Hue, Saturation and Intensity) space, whereas it is not possible in RGB space. 
3.3.2.1 HSV: Humans perceive colors as a combination of 3 colors: Red, Green and blue. The representation of HSV space is derived from the RGB space cube with the main diagonal of the RGB model as the vertical axis in HSV as shown in Fig. 11. The colors vary from unsaturated (gray) to saturated (no white component) as saturation varies from 0.0 to 1.0. Hue ranges from 0 to 360 degrees with variation from red, going through yellow, green, cyan, blue and magenta and back to red. The color space intuitively corresponds to the RGB model from which the other color models are derived through linear or nonlinear transformations.

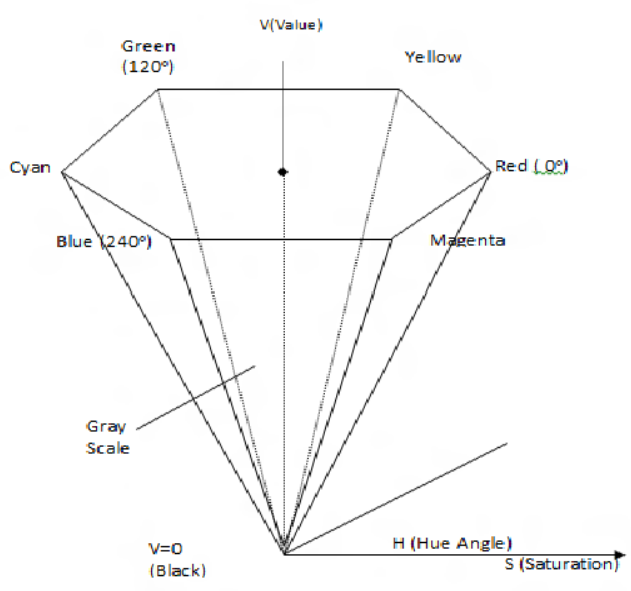

Figure 11. HSV Color Model

Based on Hue, Saturation and Intensity (HSV) color model mean and standard deviation are extracted. Mean provides average color value in the image and is given by the equation (6).

$$
\text { Mean }=\frac{1}{M N} \sum_{i=1}^{M} \sum_{j=1}^{N} P i j
$$

The hue, saturation and intensity mean generated for all kinds of field images is as shown in the Table 6. The classification accuracy for the color feature mean is as shown in the Fig.12.

Table 6 HSV Mean Values of Different Field Images

\begin{tabular}{|l|l|l|l|c|c|c|c|c|}
\hline Mean & $\begin{array}{l}\text { Brinja } \\
\mathrm{l}\end{array}$ & $\begin{array}{l}\text { Cotto } \\
\mathrm{n}\end{array}$ & $\begin{array}{l}\text { Groundn } \\
\mathrm{ut}\end{array}$ & Maize & Paddy & $\begin{array}{l}\text { Soyabea } \\
\mathrm{n}\end{array}$ & $\begin{array}{l}\text { Sugarcan } \\
\mathrm{e}\end{array}$ & $\begin{array}{l}\text { Sunflowe } \\
\mathrm{r}\end{array}$ \\
\hline Hue & 0.3183 & 0.3159 & 0.3004 & 0.2790 & 0.2630 & 0.2936 & 0.3257 & 0.2897 \\
\hline $\begin{array}{l}\text { Saturatio } \\
\mathrm{n}\end{array}$ & 0.2940 & 0.2800 & 0.3740 & 0.4720 & 0.6249 & 0.4108 & 0.2914 & 0.3153 \\
\hline Value & 0.5745 & 0.5906 & 0.5803 & 0.5609 & 0.6130 & 0.5757 & 0.6005 & 0.5795 \\
\hline
\end{tabular}




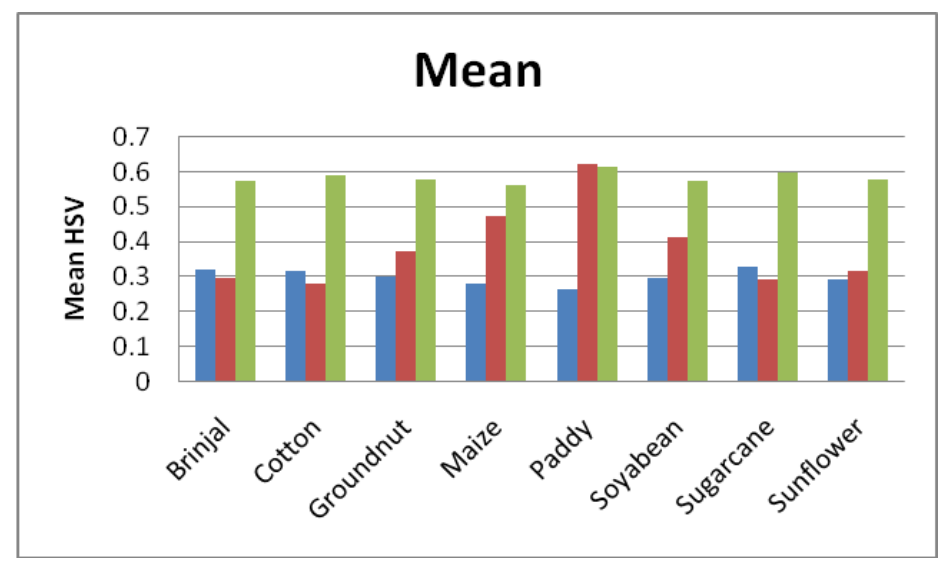

Figure 12. HSV Mean Histogram Plot

Standard deviation is defined as the square root of variance and is given by the equation (7)

$$
\begin{aligned}
& \text { Standard Deviation } \\
& \ldots \quad \text { (7) }
\end{aligned}
$$

The hue, saturation and intensity standard deviation generated for all kinds of field images and the classification accuracy is given in Table 7. Classification accuracy for the feature standard deviation is as shown in Fig.13.

Table 7 HSV Standard Deviation Values of All Field Images

\begin{tabular}{|l|c|c|c|c|c|c|c|c|}
\hline STD & Brinjal & Cotton & Groundnut & Maize & Paddy & Soyabean & Sugarcane & Sunflower \\
\hline Hue & 0.0684 & 0.0890 & 0.0786 & 0.0811 & 0.0246 & 0.0728 & 0.0721 & 0.0700 \\
\hline Saturation & 0.1265 & 0.0927 & 0.1254 & 0.2470 & 0.1198 & 0.1715 & 0.1302 & 0.1079 \\
\hline Value & 0.1995 & 0.1366 & 0.1668 & 0.1934 & 0.0808 & 0.1991 & 0.1629 & 0.1545 \\
\hline
\end{tabular}

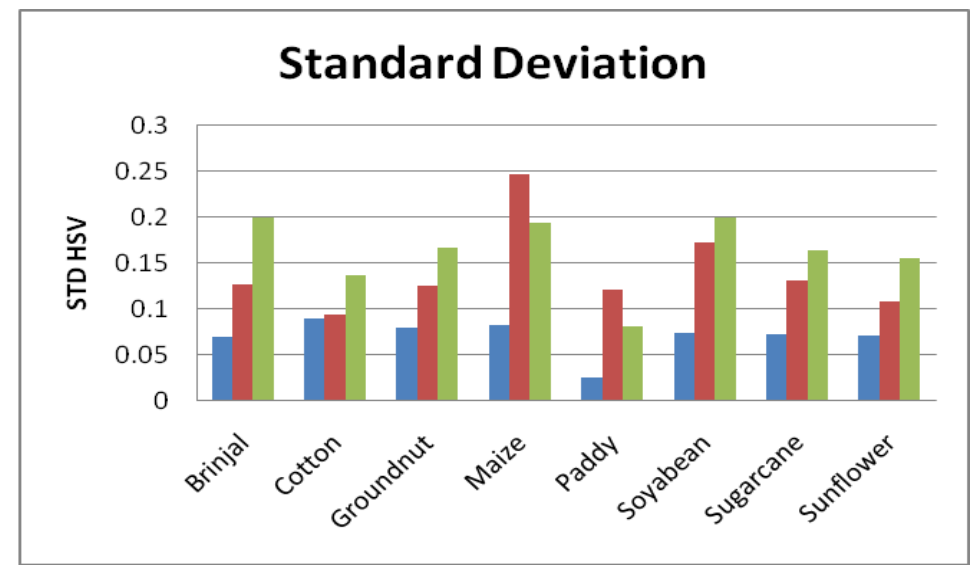

Figure 13. HSV Standard Deviation

3.3.3 Combined features: Considering individual features, the results obtained were not satisfactory. We experimented with combination of texture and color features. The results are quite encouraging. A total of seven features are finally deployed in the present work. The classification accuracy for the combined features is as shown in Fig. 14. 


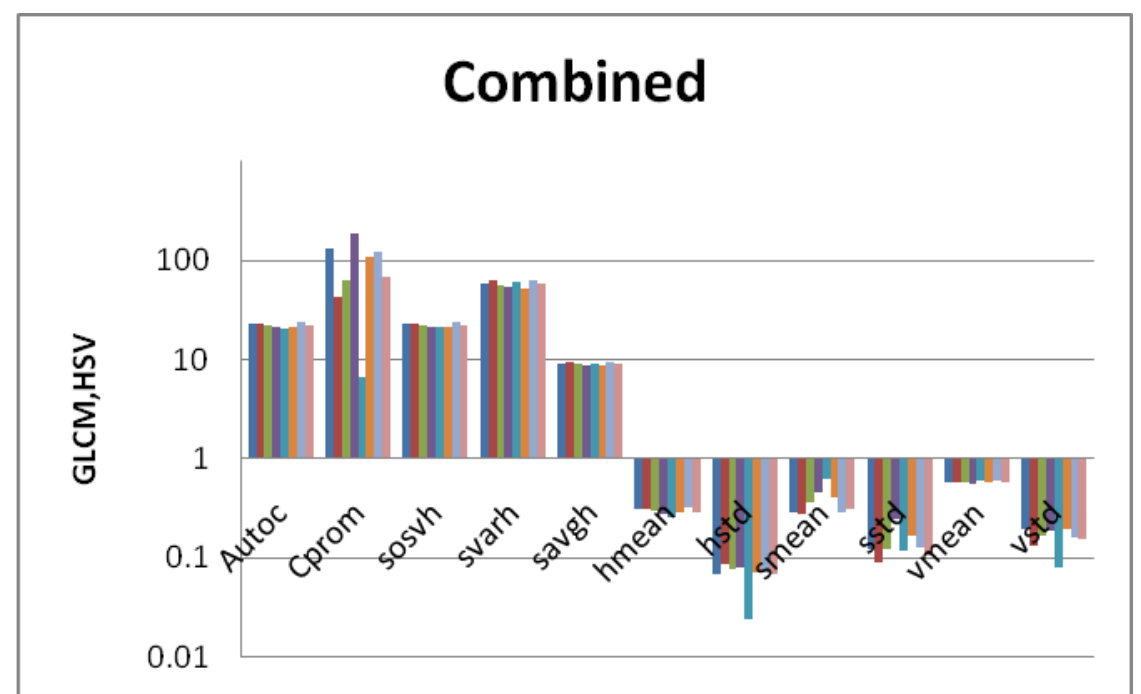

Figure 14. Classification Accuracy for Combined Texture and HSV Features

\subsection{Classification:}

Based on the computational simplicity Artificial Neural Network is used as a classifier. Feed forward multilayer network is used for the proposed work and back propagation algorithm is used for training the classifier. Classification is attempted in 3 stages, firstly, only with the texture features; secondly, with the color features and lastly with combination of both texture and color features. Input layer has seven nodes and output layer has eight nodes. The hidden layer is one. The termination error is set to 0.001 .

\section{Results and Discussion}

We have used a total of 160 images. The average classification accuracy for GLCM features is given in Fig.15. The average classification accuracy for color features is given in Fig.16. The maximum accuracy is obtained for paddy field images. The minimum accuracy is obtained for groundnut field images. This is observed for both types of features.

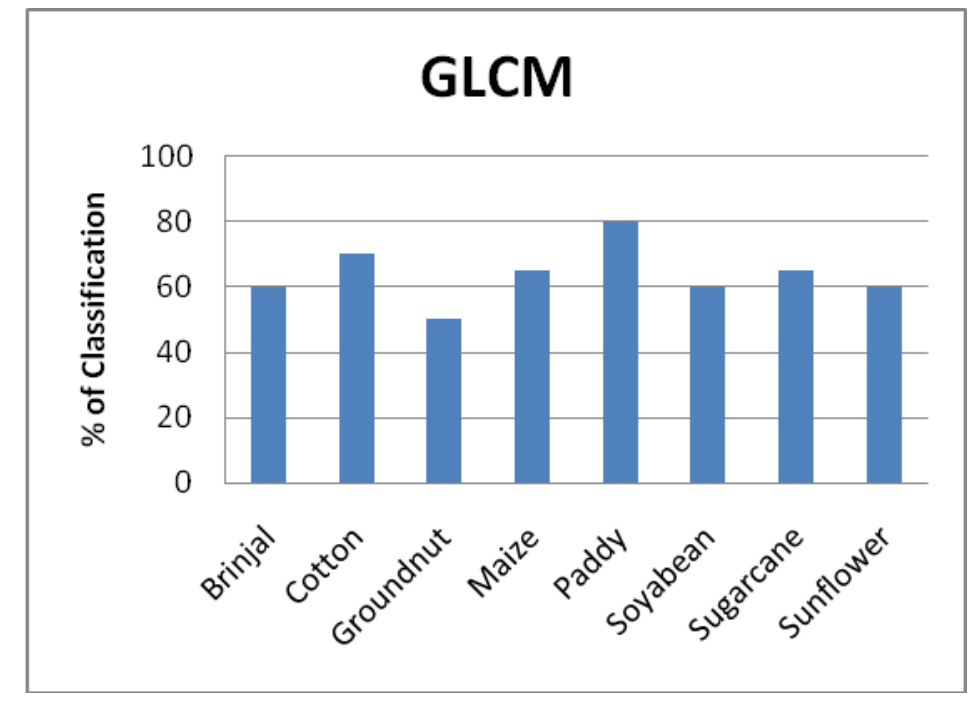

Figure 15. Classification Accuracy for GLCM Features 


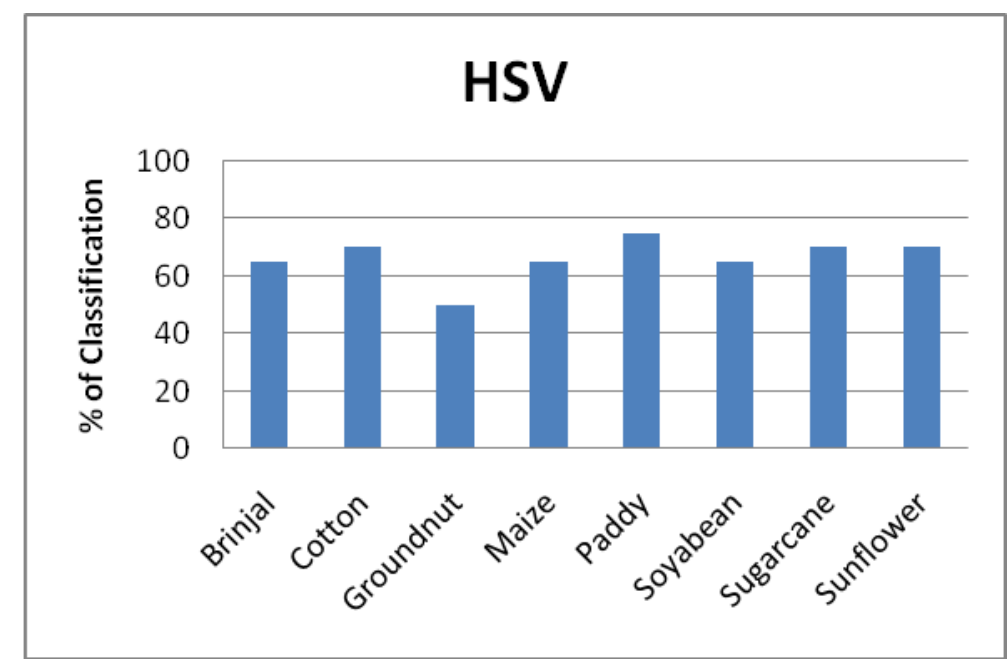

Figure 16. Classification Accuracy for HSV Features

Combination of texture and color (GLCM and HSV ) features has resulted in classification accuracy of $84.375 \%$ and is given Fig.17. Accuracies are improved for all the field images.

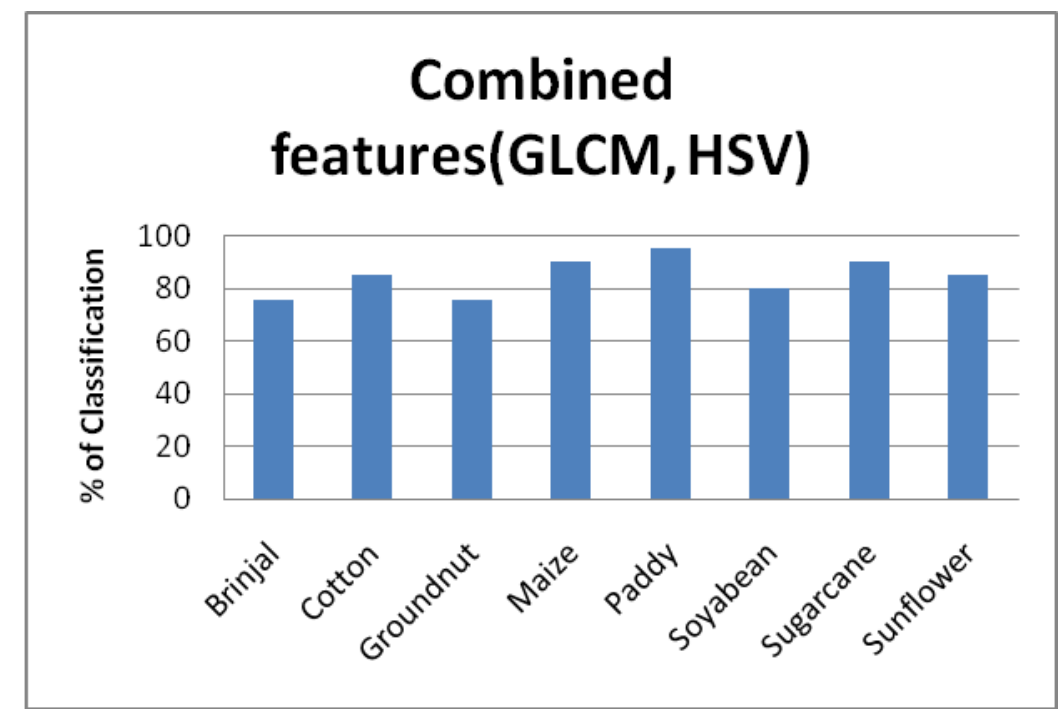

Figure 17: Classification Accuracy for Combination of Features

\section{Conclusion}

The work has reported the accuracies of classification of field images of 8 different types of crops. The color and texture features are deployed. An artificial neural network is used to classsify different types of crops. The combination of features has given good results compared to individual features. We have obtained an average accuracy of $84.375 \%$ for combination of features. The work finds application in technology deployment in Agriculture. 


\section{References}

[1] H. Syahputra,, A. Harjoko, R. Wardoyo and R.Pulungan, "Plant recognition using stereo leaf image using gray-level co-occurrence matrix", Journal of Computer Science 10, no. 4, (2013), p. 697.

[2] P. Pallavi and V. S. Veena Devi "Leaf regognition based on feature extraction and zernike moments", International journal of innovative research in computer and communication engineering", vol. 2, no. 2, (2014).

[3] G. Ariputhiran and S. Gandhimathi, "Feature Extraction and Classification of High Resolution Satellite Images using GLCM and Back Propagation Technique", International Journal Of Engineering And Computer Science, vol. 2, no. 2, (2013), pp. 525-528.

[4] V. Metre and J. Ghorpade, "An Overview of the Research on Texture Based Plant Leaf Classification", International Journal of Computer Science and Network, vol. 2, no. 3, (2013).

[5] M. Maheshwari, M. Motwani and S. Silakari, "New Feature Extraction Technique for Color Image Clustering”, International Journal of Computer Science and Electronics Engineering, vol. 1, no. 1, (2013).

[6] A.H. Kulkarni, H.M. Rai, K.A. Jahagirdar and P.S. Upparamani, "A Leaf Recognition Technique for Plant Classification Using RBPNN and Zernike Moments", International Journal of Advanced Research in Computer and Communication Engineering, vol. 2, no. 1, (2013), pp. 984-988.

[7] S. Naikwadi, N. Amoda, "Advances in image processing for detection of plant diseases", International Journal of Application or Innovation in Engineering \& Management, vol. 2, no. 11, (2013).

[8] S. Abirami, V. Ramalingam, and S. Palanivel, "Species Classification of Aquatic Plants Using PSVM and ANFIS", Pattern Recognition and Image Analysis, vol. 23, no. 2, (2013), pp. 278-286.

[9] A. Kadir, L. E. Nugroho, A. Susanto and P. I. Santosa, "Leaf Classification Using Shape, Color, and Texture Features", International Journal of Computer Trends and Technology, (2011), pp. 225-230.

[10] Bhatt, Ashutosh Kumar, Durgesh Pant, and Richa Singh. "An analysis of the performance of Artificial Neural Network technique for apple classification", AI \& society, vol. 29, (2014), pp. 103-1111.

[11] J. A. Jaleel, S. Salim, R.B. Aswin, "Artificial Neural Network Based Detection of Skin Cancer", International Journal of Advanced Research in Electrical, Electronics and Instrumentation Engineering, vol. 1, no. 3, (2012).

[12] N. Fadilah,, J. M. Saleh, H. Ibrahim, and Z. A. Halim, "Oil palm fresh fruit bunch ripeness classification using artificial neural network", In Intelligent and Advanced Systems (ICIAS), 2012 4th International Conference, (2012).

[13] R. Venkata Ramana Chary, D.Rajya Lakshmi and K.V.N Sunitha, "Feature extraction method for color image similarity", Advanced Computing: An International Journal , vol.3, no.2, (2012).

[14] H. Yu, J. Cao, W. Luo and Y. Liu, "Image Retrieval of Wood Species by Color, Texture, and Spatial", International Conference on Information and Automation, (2009).

[15] L. Zheng, J. Zhang, and Q. Wang, "Mean-shift-based color segmentation of images containing green vegetation", Computers and Electronics in Agriculture, vol. 65, no. 1, (2009), pp. 93-98.

[16] Pydipati, R., T. F. Burks and W. S. Lee, "Identification of citrus disease using color texture features and discriminant analysis", Computers and electronics in agriculture, vol.52, no. 1, (2006), pp.49-59.

[17] Fu, H., and Z. Chi. "Combined thresholding and neural network approach for vein pattern extraction from leaf images", IEE Proceedings-Vision, Image and Signal Processing, vol. 153, no. 6, (2006), pp. 881-892. 
International Journal of Signal Processing, Image Processing and Pattern Recognition Vol. 8, No. 2 (2015) 